\title{
Medical Image of the Week: Healthcare-associated Pneumonia Secondary to Aspiration
}

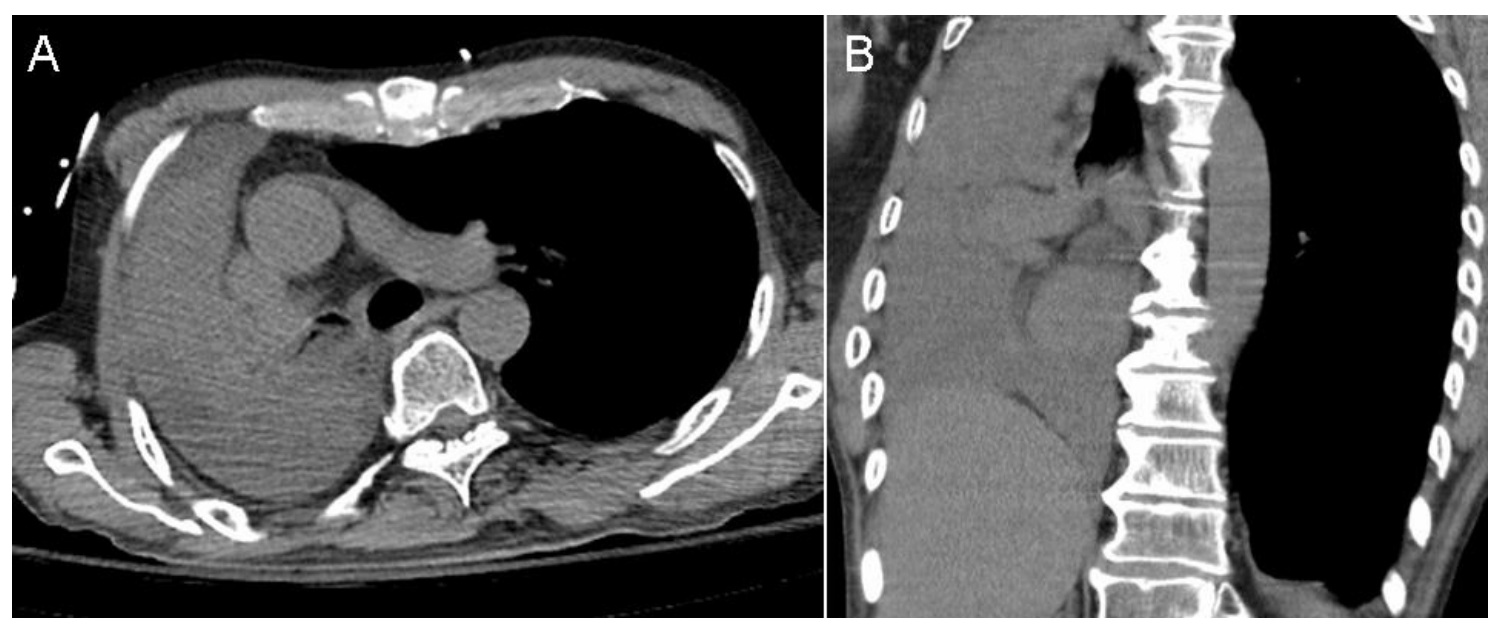

Figure 1. Panel A: Axial computed-tomography image demonstrating a foreign body within the right main-stem bronchus, with consolidation and volume loss of right lung. Panel B: Coronal view.

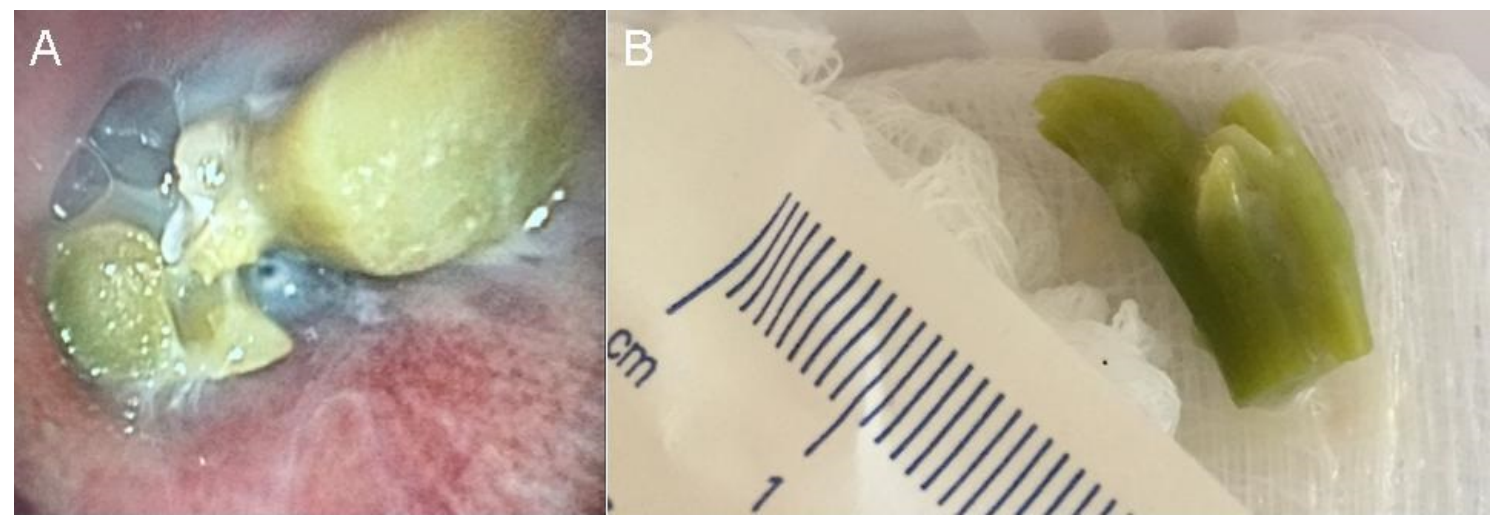

Figure 2. Panel A: bronchoscopy revealing an ingested foreign body in the right main-stem bronchus. Panel B: forceps retrieval yielded a large piece of broccoli.

A 57 year-old bedbound paraplegic man developed a worsening productive cough after being hospitalized for several days. He was brought to the radiology suite for a CT scan of the chest, revealing a soft tissue density within his right main-stem bronchus, with volume loss of his right lung (Figure 1). Bronchoscopy was performed, yielding a $2 \mathrm{~cm}$ piece of broccoli, successfully removed with forceps (Figure 2). Culture from the bronchial aspirate was positive for Pseudomonas aeruginosa. The patient's respiratory status dramatically improved after removal of the foreign body and commencement of pathogen-directed antibiotics. This study illustrates a dramatic example of healthcare-associated 
pneumonia (HCAP) secondary to aspiration, as described by the American Thoracic Society / Infectious Diseases Society of America (1).

Lavi Nissim MD, Sam Alnajjar MD and Edward Vivio RT

Phoenix Baptist Hospital

2000 W. Bethany Home Road

Phoenix, AZ 85015

\section{Reference}

1. American Thoracic Society; Infectious Diseases Society of America. Guidelines for the management of adults with hospital-acquired, ventilatorassociated, and healthcare-associated pneumonia. Am J Respir Crit Care Med. 2005;171(4):388-416. [CrossRef] [PubMed] 